\title{
Fuentes Documentales y archivo de la Residencia de Señoritas de Madrid (1915-1936)
}

\author{
María Adelina CODINA-CANET \\ mcodina@db.uc3m.es \\ Rosa SAN SEGUNDO \\ rsan@bib.uc3m.es \\ Universidad Carlos III Madrid. Instituto de Estudios de Género
}

Recibido: Julio 2015

Aceptado: Noviembre 2015

\begin{abstract}
Resumen: El centenario de la creación de la Residencia de Señoritas de Madrid 2015, es una fecha para cuestionarnos las fuentes documentales de archivo referidas para la investigación de la institución. El trabajo que nos ocupa describe del fondo documental del archivo de la institución que marcó un hito en la actividad pedagógica y en el acceso a la educación superior de las mujeres en España. La mayoría de los documentos se encuentran digitalizados, facilitándose la consulta de la base de datos para recuperar información sobre actividades y servicios que proporcionaba la Residencia. El fondo se halla instalado en 100 cajas y se estiman unos 30.000 documentos. La serie documental Correspondencia es la que contiene mayor número de documentos, más de 13.000.El estudio analiza esta serie documental por su trascendencia y proyección en los Estudios de Género y la Historia de las Mujeres. Numerosa documentación es fundamental conocerla, por lo general la descripción proporciona información sobre la gestión de la institución y las relaciones que mantenían personas influyentes en los ámbitos de la intelectualidad y la política de la época con la institución o su directora. Finalmente se describe la documentación depositada en otros centros de archivos que puede resultar interesante para la investigación
\end{abstract}

Palabras clave: Asociaciones feministas; Élites intelectuales; Estudios de género; Fuentes de documentación; Historia de las mujeres; Mujeres españolas; Residencia de Estudiantes; Residencia de Señoritas.

\section{Documentary Sources and Archives Ladies Residence in Madrid (1926-1936)}

\begin{abstract}
An aproppiate date to question the archival documentary sources to the "Residencia de Señoritas de Madrid” research, is 2015, in order to it's the centenary of its creation. The work concerned describes the archive's documentation and details the documentary collection from own archive of the institution, which marked a milestone in the educational activity and the access to higher education for spanish women. The documentary collection is digitized and provides query tools to the database to retrieve information about activities and services supplied by "Residencia". The fund is located in 100 boxes, and there are estimated 30.000 documents. The documentary series "Correspondencia" contains more than 13.000 documents. This is the series which more contains. The study examines this documentary series because of its
\end{abstract}


importance and projection at Gender Studies and Women's History. It's essential to know a lot of this documents, in order to know information about the institution managment and the relationship between influential people in intellectual areas and politicians of that time with the Institution or its director.

Finally, this work describes the diposited documentation in others archives that can be interesting for following researchs.

Keywords: Documentation sources; Feminist associations; Intellectual elites; Gender studies; Ladies Residence; Spanish women; Students Residence; Women History.

\section{INTRODUCCIÓN}

Durante el año 2015 se celebró el centenario de creación de la Residencia de Señoritas de Madrid, se conmemora una experiencia innovadora, ejemplar para la emancipación de la mujer española en el primer tercio del siglo XX, desafortunadamente truncada por la guerra civil y caída en el olvido durante los 40 años de franquismo. Un año de actos, exposiciones, congresos y tertulias que conmemoró 100 años de una práctica vanguardista en su tiempo, un pasado de conquistas que modernizó la intelectualidad y la educación superior de las mujeres, para conseguir la igualdad en el acceso a las carreras y profesiones liberales.

Sin embargo, tan solo unos años antes, en el siglo XIX, la universidad se modernizaba y las ideas liberalizadoras quedaron definitivamente asentadas con las Leyes de Claudio Moyano en 1857 (San Segundo, Codina-Canet, 2014: 8). En realidad, se partía de un contexto en que la enseñanza superior de la mujer estaba limitada en relación al varón. Pues la oferta formativaparalas más acomodadas restringía el acceso al Real Conservatorio de Música y Declamación fundado en 1830;a estudios de practicantes y matronas desde 1857 (Ley Moyano);a las Escuelas Normales de Maestras establecidas en distintas provincias españolas a partir de 1858; la Escuela de Institutrices, 1869;la Escuela de Comercio para señoras, 1878;la Escuela de Correos y Telégrafos, 1883; además de la Escuela de Enfermeras Santa Isabel de Hungría, 1896, del Instituto de Terapéutica Operativa "Instituto Rubio", 1880. En realidad aunque no existiese una prohibición explícita para obtener títulos, las aspirantes a acceder a niveles superiores de docencia topaban, más bien, con muchas dificultades entendidas como prohibiciones de carácter sociocultural, que muy pocas lograban superar. De hecho de sobra son conocidas las vicisitudes que atravesó Concepción Arenal (1820-1893) para tomar sus clases en la universidad (Santalla López, 1994).

Por lo que respecta a experiencias de instituciones consagradas a la educación superior de las mujeres, anteriores a 1915, cabe mencionar la Asociación para la Educación de la Mujer (AEM) como fruto de las ideas krausistas (Madrid, 1871), consagrada a la cultura profesional y la elevación moral y social de la mujer y posiblemente postulada como la primera iniciativa española laica de promoción de la mujer a finales del siglo XIX. De ella egresaban institutrices, tituladas en 
comercio y telégrafas. En este sentido, para Antonio Pau (2001: 119-121) "la Residencia de Señoritas es un fruto tardío de la inquietud pedagógica de Fernando de Castro promotor de la asociación”... "la Residencia no nace en el seno de la Asociación para la Educación de la Mujer, como otras instituciones de formación femenina, sino en el ámbito de otra organización krausista - la Institución Libre de Enseñanza (ILE)-. Pero no hay duda de que la Residencia de Señoritas es heredera directa de las preocupaciones, los proyectos y las ideas de Castro”. En suma, existió conexión ideológica entre ambas instituciones. De hecho por su proximidad temporal, se conocen mujeres que se sintieron atraídas por ambas instituciones, por citar algunos nombres, María Goyri de Menéndez Pidal y María Lejárraga de Martínez Sierra.

Acerca de Goyri, alumna entre 1887-1889 en la AEM, estuvo vinculada a la Residencia como profesora impartiendo enseñanza general, primaria y bachillerato para el grupo de niñas desde el curso 1916/1917 (Capel Martínez, 2009: 158), y lengua y literatura en los cursos de 1915 a 1920 (Vázquez Ramil, 2012:357-360). Y Lejárraga titulada enComercio fue contratada como profesora de inglés entre 1907 y 1909, cesando su actividad por la necesidad de tener una dedicación exclusiva a su carrera dramaturga, ya entonces reconocida internacionalmente. La obra "Canción de cuna” fue catalogada como el bestseller de su producción literaria y fue representada en teatros y en especial en los colleges femeninos de los Estados Unidos (Núñez Puente, 2008: 283-291). En la Residencia participó en tertulias culturales y pronunció conferencias.

La segunda entidad cercana a la Residencia de Señoritas es la organización International Institut for Girls in Spain(IIG), fundada en 1892 por la estadounidense Alice Gulik, una mujer emprendedora y representante de la Junta de Misiones Femeninas de la Iglesia Congregacionista, que por cierto, llevaba un tiempo en España, desde 1877 con el objetivo de instaurar la educación de alta calidad para las mujeres españolas. De hecho se las preparaba no sólo para el examen de acceso a la universidad, sino que a través de la matrícula libre, las instruían para los exámenes de licenciatura (Connelly, 1982: 197). Al fin tras algunos derroteros la institución se instaló en Madrid, el trabajo de Carmen de Zulueta (1993, 2002) profundiza este tema.

El IIG conocido también como Instituto Internacional de Miguel Ángel inició su actividad en 1903. Desde el principio existió una fuerte relación de intercambio de recursos académicos entre instituciones y también con el Instituto-Escuela (1918), el proyecto pedagógico que trasladó los principios de la ILE: laicidad y coeducación, a la educación secundaria. Finalmente, cabe mencionar una tercera institución relacionada y ubicada en Barcelona cuya meta estuvo dirigida a mejorar el bienestar intelectual y profesional de las mujeres, el Institut de Cultura i Biblioteca Popular per la Dona (Barcelona 1909-1936). Fue un centro de cultura y formación laboral cuya fundadora y directora Francesca Bonnemaison mantuvo una 
comunicación formal con María de Maeztu, de hecho intercambiaron correo durante más de diez años.

Tras esta introducción contextual, el estudio que nos ocupa es la recopilación de fuentes documentales de archivo para investigar temas, instituciones o personas relacionados con la Residencia de Señoritas (1915-1936). El objetivo es describir el fondo documental del archivo histórico generado durante su actividad, complementado con la documentación obtenida en otros archivos españoles. Identificando fuentes de documentación para investigaciones encauzadas a la contribución de las mujeres en el proceso histórico de la ciencia y la sociedad y facilite futuras líneas de investigaciones historiográficas, sociológicas y antropológicas.

\section{METODOLOGÍA}

La estrategia para identificar las fuentes documentales plantea conocer las funciones técnico-administrativas de la institución y las relaciones funcionales y orgánicas con otras instituciones en el transcurrir de su existencia. Las instituciones satélite de la Residencia de Señoritas consideradas son la Junta de Ampliación de Estudios e Investigaciones Científicas JAE o JAEIC (1907), el International InstituteforGirls in Spain (1903), la Residencia de Estudiantes (1910) y el Instituto-Escuela (1918).

Se aporta una descripción y análisis crítico de la Residencia como institución en la historia de las mujeres, para transmitir más bien la importancia de los documentos conservados en su archivo.

El método de trabajo se basa en la descripción las fuentes documentales primarias para la investigación, recopilando recursos de información de apoyo a los estudios cuya perspectiva historiográfica es la contribución de las mujeres en la historia.

Se analiza el archivo original de la Residencia, documentación originada en el desempeño de sus actividades y se recopilan fondos localizados en los siguientes archivos institucionales: Archivo de la JAE, Archivo de la Residencia de Estudiantes, Archivo General de la Administración y Archivo Central de Educación. Respecto al Archivo del Institute International no tuvimos acceso ya que según fuentes de la misma institución el archivo fue cedido hace unos años a Smith College, se encuentra en Northampton, Massachusetts (USA).

Quedan excluidos del estudio los archivos privados o familiares de personas relacionadas con la Residencia, ya que quedaba fuera del alcance de este estudio. Sin embargo, reconocer que las fuentes no incluidas podrían contener informaciones de un valor inestimable, pero excede del propósito de esta investigación.

Por lo general, cuando se abarca el estudio de fuentes documentales de archivo del feminismo, se echa en falta el acceso a un censo de archivos feministas y de archivos personales de activistas feministas en España, en particular facilitaría esta tarea "la creación de una guía de fuentes para los estudios de género y la historia del 
movimiento feminista (Codina-Canet, San Segundo, 2015:17). En realidad el estudio de las fuentes documentales de archivo es un trabajo todavía pendiente para el feminismo académico.

\section{LA RESIDENCIA DE SEÑORITAS EN LA HISTORIA}

La Residencia de Señoritas de Madrid, fue el primer centro oficial estatal destinado a albergar mujeres universitarias o que preparasen su ingreso en Facultades, Escuela Superior de Magisterio, Escuela Normal, Escuela del Hogar, el Conservatorio Superior de Música o cursos de Biblioteconomía (JAE, 1916: 300).

Su creación en 1915, se engloba en el proyecto pedagógico cuyo objetivo general era transformar la sociedad por medio de la educación y la cultura de las élites de la sociedad liberal-burguesa. De hecho es uno de los centros del proyecto institucionalista de la JAE, por consiguiente, la relación de ambas instituciones fue de dependencia orgánica administrativa.

Durante 21 años de actividad se producen en España varios procesos de transformación política, que culminan en cambios en el sistema de gobierno central, pasando de un gobierno liberal a una Dictadura por Real Orden (1923-1930) y al poco tiempo a la subsiguientemente II República (1931-1939). La Guerra Civil iniciada en 1936 fue el motivo de cese de la actividad de la Residencia.

No obstante, para dar con el origen de la idea de creación, hay que remontarse incluso unos años atrás, cuando el pedagogo e ideólogo Giner de los Ríos, heredero de las ideas krausistas del XIX (Sanz del Río y Fernando de Castro), junto con Cossío y Castillejo impulsaran el proyecto pedagógico de la JAEIC. Y definitivamente el ideario de la ILE consiguiera materializarse en 1907 con apoyos de políticos liberales de la talla de Segismundo Moret y el Conde de Romanones, de científicos como Ramón y Cajal, y Negrín.

La reorganización del sistema se plasmó en 1910 abordando tanto el ámbito pedagógico como el de la investigación científica. Ese mismo año se crearon el Centro de Estudios Históricos, el Instituto Nacional de Ciencias Físico-Naturales; en años sucesivos la Comisión de Investigaciones Paleontológicas y Prehistóricas, el Laboratorio de Química (1912); el Instituto Español de Oceanografía (1914);el Laboratorio y Seminario Matemático de Julio Rey Pastor (1915);la Junta de Parques Nacionales, el Laboratorio de Fisiología y Anatomía de los Centros Nerviosos (1916); el Laboratorio de Histología Normal y Patológica (1919);el Centro Nacional de Investigaciones Biológicas (1920); y años más tarde el Instituto Nacional de Física y Química (1932). En suma, estas instituciones de investigación de nueva creación dan idea de la magnitud del proyecto desarrollado a principios de siglo en España.

En el ámbito pedagógico, la Residencia de Estudiantes (1910)fue la primera fase del proyecto dedicado a varones, es decir, cinco años después quedaría completado 
con la Residencia de Estudiantes“Grupo de Señoritas” así se le denominó también, un proyecto único que representó un firme impulso a las políticas de igualdad en el ámbito educativo (Cabrera Calvo-Sotelo, 2008: 216).

En todo caso, aclarar que el término igualdad de la cita, queda referido a la circunscripción de género dentro de la clase social liberal-burguesa con posibilidades económicas para el acceso a los estudios superiores. No obstante, la lucha por la igualdad de género tanto a finales del siglo XIX como en el primer tercio del XX fue un axioma considerado interclasista. Por tanto, la igualdad social se circunscribe a movimientos sociales y sindicales, en donde las mujeres mantuvieron una participación política colectiva, aunque el protagonismo de ellas, por los condicionantes de género del momento se encuentre todavía invisibilizado (Nasch, 2009: 117).

Por lo que respecta al ideario de la Residencia, para Vázquez Ramil (2012: 320) confluyeron varios fines "la Residencia se constituyó como institución residencial, centro académico, aula de cultura y sede de la rama internacionalista del movimiento feminista”, su análisis desde la perspectiva de género de la institución analiza la transcendencia histórica, de hecho, la relación de dependencia administrativa con la Residencia de Estudiantes. En su trabajo, Vázquez incorpora una relación nominal de las residentes durante 1915-1936, en particular, construida a partir de registros y memorias del archivo.

En cuanto a las características del aforo académico, destaca la tendencia creciente entre las residentes matriculadas en la universidad en los decenios 20 y 30.

Según observa, las alumnas experimentan un acusado incremento, pasando de un $17 \%$ en el curso $1915-1916$ a un 71\% en 1933-34. En otro estudio, realizada la comparativa con los varones residentes, en ese mismo curso, se contabilizan 150 varones, frente a 250 mujeres, así pues, se constata un considerable aumento del número de alumnas, por tanto estos datos señalan el grado de cohesión de aforo de residentes universitarios en ambas instituciones (Magallón Portolés, 2004: 168-170).

Por otra parte, en relación a las actividades académicas que se impartieron, se mencionan cursos de lenguas inglesa, francesa y alemana, impartidos por nativas; y otros servicios, de laboratorio de química, biblioteca; y clases complementarias. Vázquez incluye una relación de asignaturas, cursos y profesorado generalmente femenino encargado de los mismos.

En cuanto a la consideración de aula de cultura, cabe mencionar las conferencias y tertulias con la intelectualidad de la época. Entre las personalidades del movimiento intelectual que dieron conferencias cuenta un elenco de mujeres como son: Clara Campoamor, María Montessori, Gabriela Mistral, Victoria Ocampo, María Lejárraga, Concha Méndez, Victoria Kent entre otras; y Luis de Zulueta, Ramón Gómez de la Serna, Rafael Alberti o Federico García Lorca entre las decenas de intelectuales que participaron en las actividades de la Residencia. Así pues, este ambiente permitió a la institución consolidarse como un centro de recepción e intercambio vanguardista. 
El innovador programa de la Residencia fue conducido por María de Maeztu, su directora junto a sus colaboradoras más cercanas Eulalia Lapresta y Rafaela Ortega y Gasset.

Aparte, cabe mencionar que Maeztu fue impulsora de otras asociaciones feministas como el Lyceum Club Femenino (1926) y la asociación Juventud Universitaria Femenina (1920), con representación en la International Federation of UnviversityWomen. La citada asociación organizó el Congreso Internacional de Mujeres Universitarias celebrado en 1929 en Madrid, alojándose buena parte de las asistentes extranjeras en la Residencia (Millard, 1990: 11-13).

En cuanto alLyceum Club Femenino Español se constituyó como centro de ocio, cultura y auxilio femenino, reconvirtiéndose en poco tiempo en una asociación cultural orientada a la defensa de la igualdad femenina (Eiroa, 2015: 3). Tanto la Residencia como el Lyceum, con más de 400 asociadas (Aguilera Sastre, 2011: 77), fueron instituciones que se convirtieron en referentes para las mujeres que alcanzaron protagonismo en la intelectualidad española en los años veinte y treinta. En consecuencia, gracias a las relaciones institucionales, el archivo conserva documentación de todas las entidades mencionadas.

Finalizamos esta exposición, sumándonos al análisis crítico de exclusión por la situación de desigualdad de las mujeres en el tratamiento de la asignatura de Historia en los libros de texto. A pesar de numerosas investigaciones sobre esta cuestión y algunos éxitos notables en esta dirección (Fagoaga 1985 y 2002), (Pozo Andrés, 1985), (Hurtado, 1999), (Mangini, 2001, 2006), (Kirkpatrick, 2003), (Simón Palmer, 2006), (Marina; Rodríguez de Castro, 2009), (Alcalá Cortijo et alt., 2009), (Ribagorda, 2009), (Pérez-Villanueva Tovar, 1989, 1990, 2011), considérese esta relación orientativa, sin ánimo de ningunear otras investigaciones que aportan conocimiento de la historia liberándolo de los sesgos de género en esta etapa de nuestro pasado. Para ampliar bibliografía se podría consultar Barnciforte (2015) el estudio sobre el prolífico enfoque biográfico y las numerosas líneas historiográficas del feminismo abiertas sobre estas décadas por el feminismo académico.

Sin embargo, las mujeres siguen invisibilizadas o silenciadas o son puntos ciegos en los manuales de historia y literatura publicados en España, mujeres que destacaron tanto como los varones en el panorama intelectual y artístico de la Edad de Plata de la cultura española. En qué lugar quedaron Marga Gil Roësset, Concha Méndez, María Zambrano, María Teresa León, Rosa Chacel, Josefina de la Torre, Maruja Mallo, María Lejárraga o Ernestina de Champourcin, entre otras. Preexisten mujeres vanguardistas y modernas del primer tercio del siglo XX, cuya existencia y relatos de vida se conocen en el feminismo académico, pero no trasciende a la sociedad y son todavía asignatura pendiente para la historia, la cultura y la ciencia.

Las mujeres que lucharon por la emancipación de esta época son un ejemplo del "eslabón perdido" o la llamada generación perdida (Gómez Blesa, 2015), cuyas biografías están siendo recuperadas en estos últimos años. Según Pérez Sendeño sobre la historia de las mujeres "Los numerosos testimonios que las protagonistas 
dejaron por escrito (Arbós, Burgos, Campoamor, Méndez, Oyarzabal, Nelken, Kent, Arnáiz, Falcón, Tejero, etc.), refleja cómo eran conscientes de estar haciendo historia y también de que era necesario contarlo” (Alcalá Cortijo, 2009: R.16).

La descripción de la Residencia en la historia, es en suma incidir en la importancia de esta institución, en la recuperación y la reconciliación de la memoria de las mujeres en el seno de la sociedad. Y el estudio de los fondos de archivo como fuente historiográfica es fundamental para recuperar, investigar y profundizar en la memoria de las mujeres olvidadas, exiliadas y depuradas.

\section{ARCHIVO DE LA RESIDENCIA}

El archivo de la Residencia de Señoritas es un corpus documental cuyo origen está generado en el ejercicio de las competencias y funciones administrativas de la institución gestión económica, personal y académica. La descripción permite conocer el tipo de actividades, de servicios y de personas vinculadas a la Institución, además existe documentación ajena a la gestión administrativa propia, como consecuencia del uso transitorio de los locales como internado de niñas del Instituto-Escuela.

El archivo con un siglo de existencia se encuentra integro, las fechas extremas de los documentos se sitúan entre 1915 y 1938. Además presenta un excelente estado de conservación y la documentación es única, esta naturaleza genuina le aporta un valor añadido, por tratarse de documentación irrepetible.

Por consiguiente, en la actualidad constituye un patrimonio documental de la historia de la educación y la cultura de las mujeres y un referente de consulta para la investigación histórica y sociológica del primer tercio del siglo XX.

El archivo cuenta con la particularidad de haber permanecido extraviado durante cincuenta años y tras ser rescatado del olvido, es decir se encuentra a disposición pública desde el año 1987 (Zulueta; Moreno, 1993:11) y hace unos años se halla digitalizado. En cualquier caso, se trata de una fuente documental poco citada en investigaciones, y paradójicamente aun conteniendo información generada hace cien años es un archivo reciente con muchas posibilidades para la investigación futura.

El archivo se encuentra depositado en la Fundación Ortega-Marañón junto con la biblioteca y los archivos personales de José Ortega y Gasset (1883-1955) y Gregorio Marañón (1887-1960). Con todo, a diferencia de estos, no está considerado oficialmente un "Bien de interés cultural", sin embargo no le faltan méritos para conseguir dicha mención considerando su peculiar naturaleza y características históricas mencionadas. 


\subsection{EL FONDO DOCUMENTAL}

El fondo contiene toda la documentación de la gestión administrativa de la Residencia y del grupo de niñas del Instituto-Escuela. Pues Maeztu pertenecía a la junta directora del Instituto, de ahí que se conservan en el archivo documentos asociados a esta institución académica. El fondo se encuentra instalado en 100 cajas, se estiman unos 30000 documentos, prevaleciendo la ordenación cronológica y alfabética.

Se observa la ausencia de instrumentos de descripción documental normalizada como inventario, catálogo y cuadro de clasificación del fondo, sólo encontramos accesible un índice topográfico de la instalación.

Por lo que parece, inicialmente los trabajos archivísticos realizados entre 1984 y 1987 consiguieron ordenarlo y clasificarlo (López Cobo; Basabe, 2007), posteriormente se realizaron varios proyectos de clasificación. Sin embargo, nos comentaba el personal del archivo que la documentación está pendiente de un estudio a fondo y se precisa continuar con esta tarea archivística de clasificación y descripción.

Se adjunta el índice topográfico del fondo documental.

\subsection{SERIE CORRESPONDENCIA}

El fondo epistolar es la serie de mayor documentación, por lo general esta sección contiene cartas autógrafas y escritas a máquina, originales, copias de misivas, y en menor volumen telegramas y postales, ordenado cronológicamente. Las fechas extremas son 1915-1936, y el volumen asciende a más de 13000 documentos, distribuidos en 42 cajas de instalación y cerca de 1900 carpetas. Esta parte de fondo epistolar podría ser una interesante fuente de información para estudios sobre la historia social de la cultura escrita en diferentes ejes temáticos, en torno al análisis de la escritura, a los discursos, a las prácticas y a las representaciones de dichos testimonios escritos. Además para profundizar sobre las relaciones y los procesos de comunicación de la Residencia con representantes de otras instituciones, con alumnas y familiares, y con personalidades coetáneas que mostraban interés por la Residencia o participaban en sus actividades.

Tras la ordenación del fondo, la serie constituye casi la mitad del caudal documental, la instalación se encuentra parcelada en cuatro subseries.

En cualquier caso la documentación se pueden consultar íntegramente en formato digital. De hecho el manejo de la base de datos permite filtrar la consulta, algunos datos muestran la capacidad y potencia en recuperar resultados. Por poner varios ejemplos se practicaron búsquedas por ciudades, en el campo de búsqueda lugar de origen o procedencia de las cartas y la cantidad de documentos recuperados fueron estos.

Las ciudades consultadas fueron: París (371 documentos), Nueva York (190), Londres (147), Berlín (26), Boston (15), Washington (11), Roma (5), Dresden (1); 
por origen de ciudades españolas Madrid (3825), Bilbao (2825), Barcelona (591) y Valencia (353).

La instalación de la Correspondencia se encuentra dividida en cuatro subseries.

- María de Maeztu

. Notables

. ALV-ZW

. Ordenada por años

- Subserie María de Maeztu

La documentación se encuentra instalada en la caja 19, contiene 300 misivas ordenadas cronológicamente con fechas extremas 1917-1933. Se trata de copias de cartas escritas por Maeztu despachando numerosos asuntos tanto personales como concernientes a la actividad de intendencia de la Residencia y del Instituto-Escuela. Los destinatarios son personas físicas o empresas. Y otras cartas manuscritas dirigidas a Eulalia Lapresta secretaria de Maeztu escritas durante sus estancias en el extranjero. Algunas de ellas conservan estampado el membrete impreso del Hotel o Institución donde se alojaba. Algunos ejemplos: Bern SuisseParln, hotel Favorite (septiembre 1925), Grand Hotel du Louvre (Mayo 1923), A Bord de "France" Trasatlantique (Junio 1923), Institución Cultural Española (Buenos Aires, agosto 1926), hotel Place, Vapor Correo Reina Victoria Eugenia de la compañía trasatlántica de Barcelona (Tenerife, mayo 1926), Columbia University in the Cup of New York: Departament of Romance Languages (Julio 1923), Mulrmomah Hotel Portland Oregon (Julio 1923), Continental Hotel Cauterets (Agosto 1923), Colonial Hotel San Francisco (Julio 1925).

Se destaca un documento fechado en el año 1917, de 8 cuartillas autografiadas titulado Biblioteca escolar en la que se describe la organización de una biblioteca escolar.

- Subserie Correspondencia Notables

Esta serie facticia está instalada en dos cajas y contiene cartas dirigidas a la directora, la mayoría son manuscritas, algunas de ellas escritas sobre papel con logotipo impreso.

Desconocemos los criterios aplicados en las tareas de clasificación, no se han encontrado referencias, intuimos que en su momento se tomaron acertadas decisiones para valorar los documentos y quedaron agrupados en esta subserie.

Las cartas "Notables" son una documentación fundamental y en general los escritos custodiados proporcionan información sobre las relaciones mantenidas con las élites de la intelectualidad, la política, comerciantes e industriales de clases medias y altas; y aristócratas. En cuanto a la tipología documental incluye cartas, postales y telegramas de contenido variado, en algunos casos existen documentos fechados durante todo el período de vigencia de la institución. Por ejemplo de Rafaela Ortega y Gasset colaboradora de María de Maeztu, Alberto Jiménez Fraud 
director de la Residencia de Estudiantes o Ramiro de Maeztu hermano de la directora. El fondo resulta una fuente de información interesante para completar datos biográficos (Egoscozábal Carrasco y Mediavilla Herreros, 2012).

El listado de Notables comprende 160 nombres, la relación entre mujeres y varones es 1:5, es decir por cada mujer hay cinco varones, así pues, está claro que la disposición está completamente masculinizada.

Por una parte, las mujeres incluidas son: Sra de Azaña; María Baeza; Clara Campoamor; Zenobia Camprubi; Sofía Casanova Lutostawska; Carmen Conde; Carmen de Cossío; Natalia Cossío de Jiménez; Marie Curie; Vda. de Decroly; Concha Espina; Gloria Giner de los ríos; María Goyri; Victoria Kent; Eulalia Lapresta; María Teresa León; Anita de Maeztu; Ángela de Maeztu; Mabel de Maeztu; Jimena Menéndez-Pidal; Gabriela Mistral; María Moliner; María Montessori; Margarita Nelken; Soledad Ortega Spottorno; Rafaela Ortega y Gasset; Mr. E. Allison Peers; Alice Pestaña; Rosa Spottorno de Ortega; Alfonsina Storni; Juana (Vda. De Maeztu) Whitney; además de María Zambrano.

En cambio, por la otra, hay 130 varones vinculados a alta política, intelectualidad, ciencia, universidad, y otros tantos con un status social elevado. En todo caso sería excesivo nombrarles a todos, de ahí que en aras de la prudencia se reduce el listado, que más bien sirva como ejemplo ilustrativo: Corpus Barga; Domingo Barnés; José de Castillejo; Alberto Jiménez Fraud; Mariano Benlliure; José Calvo Sotelo; Santiago Casares Quiroga; Manuel Bartolomé Cossío; Ramón Gómez de la Serna; Ramón Menéndez-Pidal; Ramiro de Maeztu; José Ortega y Gasset; Julio Rey Pastor y Conde de Romanones.

Por consiguiente, analizando los datos desde una perspectiva de género, esta proporción 1:5 de personalidades resulta de sospechosa desigualad, en consecuencia se pone en observación la revisión de criterios utilizados para la descripción.

- Subserie Correspondencia ALV-ZW

La correspondencia ALV-ZW contiene el correo recibido por la institución, se trata de cartas originales instaladas en 27 cajas, son cartas enviadas por personas que mantuvieron relación con la Residencia, se trata en general de alumnas y familiares, sin embargo se encontraron personajes que han trascendido en uno u otro campo en la historia de la sociedad.

Por poner un ejemplo de encontramos en esta subserie a mujeres trascendentes en sus campos de desarrollo como Francesca Bonnemaison, feminista fundadora de la Biblioteca Popular de la Dona y Escola de la Dona de Barcelona, a Luisa Cuesta feminista, investigadora y escritora, a Matilde Huici activista feminista, jurista y política, a Margarita de Mayo Izarra, escritora, a Victoria Ocampo, escritora y editora, y María Sánchez Arbós, pedagoga. 
Las cartas se ordenan alfabéticamente por carpetas: C. AB - ALV (83 carpetas),ALV - AZ (84), BAC - BAS (59), BAS - BORR (83), BORR - CAR (78),CAR - CLA (85), CLA - CUY (63),CHA - DUT (73),EA - FERN (76),FERN - FY (48), GAB - GAS (71), GAS - GW (95), HAD - IZ (69), JAC - CKY (50), LAB - LOW (73), LOW - MAR (62), MAR - MON (75), MON - NY (64), OBE-PELL (42), PELT-QUI (58), RAG-RIU(67), RIU-RUB (75),SAA-SAN(58), SANZ-SIM(35), SIM-SW(31), TAB-VALL (54), VALL-ZW(68).

- Subserie Correspondencia ordenada por años

Se trata de correspondencia ordenada por series temporales de 1920 a 1936, contiene copias de cartas despachadas por la dirección, o por encargo de ella. Nos llamó la atención no encontrar en esta sección, documentos producidos en años anteriores. La suma total asciende a 6557 documentos instalados en 11 cajas de la caja 50 a la 59, ordenadas progresivamente por año y mes.

Tabla1. Correspondencia ordenada por años

\begin{tabular}{|l|c|c|c|}
\hline \multicolumn{1}{|c|}{ Instalación } & Año & $\mathrm{N}^{\circ}$ DOC. & Signatura \\
\hline \multirow{2}{*}{ Caja 50: Correspondencia 1920-1922 } & 1920 & 125 & S. 50/01-10 \\
& 1921 & 431 & S. 50/11-21 \\
& 1922 & 354 & S. 50/22-32 \\
\hline Caja 51: Correspondencia 1923 & 1923 & 327 & S. 51/01-08 \\
\hline \multirow{2}{*}{ Caja 52: Correspondencia 1924-1925 } & 1924 & 411 & S. 52/01-08 \\
& 1925 & 496 & S. 52/09-14 \\
\hline \multirow{2}{*}{ Caja 53: Correspondencia 1926-1928 } & 1926 & 206 & S. 53/01-10 \\
& 1927 & 281 & S. 53/11-21 \\
\hline Caja 54: Correspondencia 1929 & 1928 & 326 & S. 53/22-33 \\
\hline Caja 55: Correspondencia 1930 & 1929 & 825 & GS. 54/01-12 \\
\hline Caja 56: Correspondencia 1931 & 1930 & 761 & S. 55/01-12 \\
\hline Caja 57: Correspondencia 1932 & 1931 & 638 & S. 56/01-12 \\
\hline Caja 58: Correspondencia 1933 & 1932 & 621 & S. 57/01-12 \\
\hline & 1933 & 444 & S. 58/01-12 \\
Caja 59:Correspondencia 1934-1936 & 1934 & 305 & S. 59/01-32 \\
& 1935 & 152 & S. 59/12-22 \\
& - & 54 & S. 59/23-32 \\
\hline
\end{tabular}

El volumen de documentación expedida por año es variable, así como el asunto despachado. Se desconoce su ordenación original pero se trata por lo general de copia de correspondencia, en papel de tipo perforado. Los documentos no presentan anotaciones originales de una anterior clasificación, como por ejemplo ordenadas por el tipo de actividad o servicio. 
Se realizó un recuento manual de las cajas, el período más productivo de la institución se genera en las series cronológicas que van desde 1929 a1932. Se observa que el mes anual de mayor despacho de correspondencia es septiembre, este incremento se justifica por entenderse mayor fluidez informativa al coincidir en septiembre el período de matrícula de las residentes y el comienzo del curso académico.

La tabla muestra el número de documentos disponibles de cada año y su correspondiente signatura topográfica.

\section{DIGITALIZACION DEL FONDO}

La digitalización del archivo se encuentra en estado avanzado, el proyecto de digitalización es un proyecto de investigación en curso y con la preservación documental se conseguirá la pervivencia de unos materiales particularmente frágiles. El fondo digitalizado está en proceso de descripción y los documentos digitalizados convertidos a formato Portable Document Format (pdf) están volcados al programa gestor de datos Knosis Blue. La base contiene actualmente 19 269 documentos, las Series digitalizadas son Administración, Invitaciones y Correspondencia, contiene 13439 registros de cartas. Los años de mayor actividad documental son 1929 que acumula más de mil y 1925 se acerca al millar de documentos. El cuadro muestra cifras provisionales, que variarán al finalizar la incorporación de documentos digitalizados.

Tabla 2. Número de documentos digitalizados por año

\begin{tabular}{|c|c|c|c|c|c|c|c|c|c|c|c|c|}
\hline Año & 1915 & 1916 & 1917 & 1918 & 1919 & 1920 & 1921 & 1922 & 1923 & 1924 & 1925 & 1926 \\
\hline $\mathrm{N}^{\circ}$ Dc. & 50 & 360 & 637 & 409 & 336 & 450 & 629 & 629 & 679 & 892 & 980 & 650 \\
\hline Año & 1927 & 1927 & 1929 & 1930 & 1931 & 1932 & 1933 & 1934 & 1935 & 1936 & 1937 & 1938 \\
\hline $\mathrm{N}^{\circ}$ Dc. & 635 & 624 & 1078 & 857 & 649 & 772 & 695 & 516 & 277 & 150 & 0 & 0 \\
\hline
\end{tabular}

La descripción previa de los documentos y el valor de cada campo es esencial para la recuperación documental, actualmente el proceso técnico de registros se encuentra en una etapa de pre-catalogación documental, sería deseable que al módulo se añadiesen algunos campos y se completase con una información catalográfica más detallada.

Los términos descriptores utilizados en la indización son palabras en lenguaje natural, por tanto carece de listado controlado de términos, tesauro o listado de términos autorizados. Los descriptores que se utilizan son onomásticos, cronológicos y geográficos; el descriptor temático no ha sido incorporado. Detectamos la ausencia de instrumentos de descripción más completos como catálogos, inventarios, cuadro de clasificación o listado de términos controlados que posiblemente limitan la recuperación de datos.

Aunque, el proyecto de descripción se encuentra inconcluso, sin embargo, la base 
se puede consultar en la sede de la Fundación Ortega y Marañón donde se custodia.

El resultado de la digitalización son documentos convertidos en imagen, por lo que no reconoce el texto del cuerpo de los documentos.

La base de datos integra herramientas de consulta mediante un lenguaje de interrogación basado en palabras o grupos de palabras solas o combinadas y la recuperación documental se asienta en la búsqueda por términos o valores descritos en cada campo. Técnicamente la opción de carga de términos de los valores de los campos en un índice está desactivada por lo que no se puede consultar y utilizar como referencia de búsqueda, disminuyendo la capacidad de interrogación del archivo digital. De los 250 campos de descripción permitidos en Knosis Blue se utilizan 22, la imagen capturada muestra el título de los campos de descripción.

Figura 1. Captura de pantalla del buscador de la base de datos

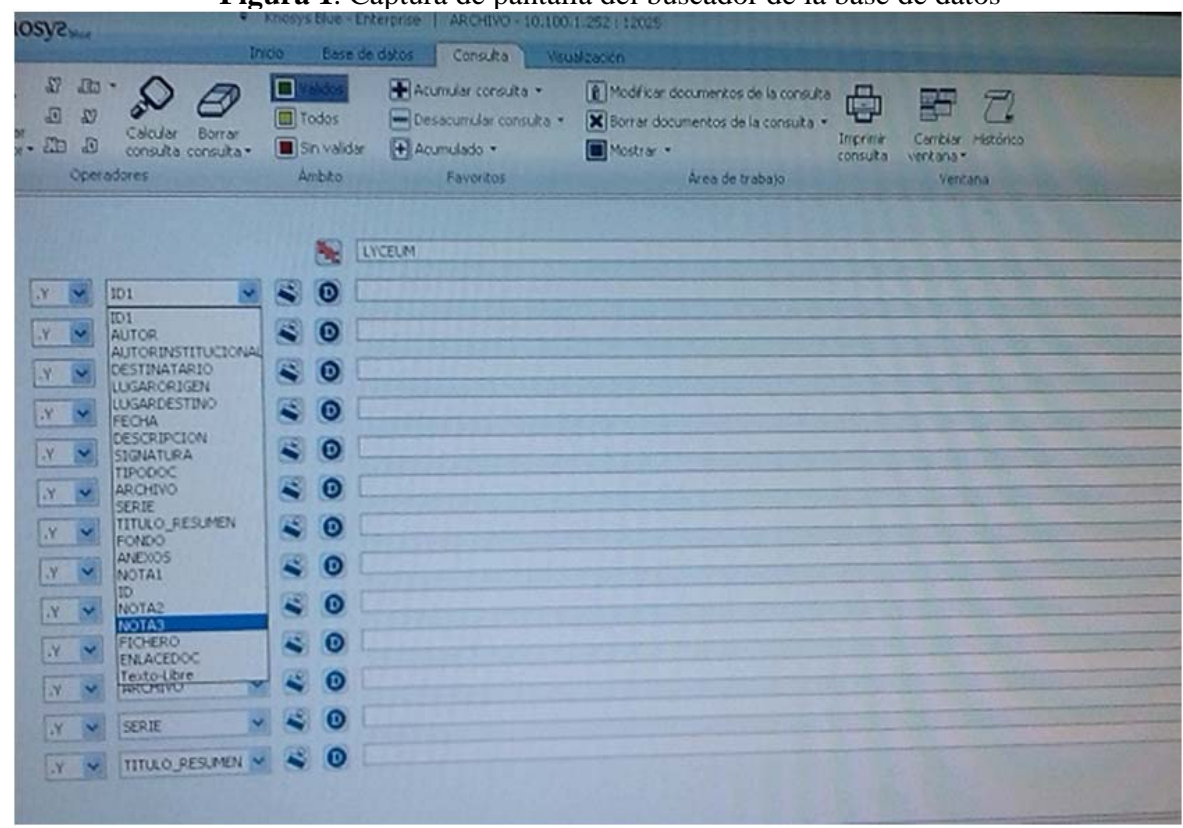

\section{OTROS ARCHIVOS}

\subsection{ARCHIVO DE LA JAE}

Tanto el archivo como la biblioteca de la antigua JAE, son el fruto de una larga labor de rescate de numerosos legados que permanecían dispersos y en gran parte inaccesibles para los investigadores, con el objeto de reunirlos y ponerlos al alcance de los investigadores, en un proceso que sigue abierto y promete nuevas incorporaciones. 
Con un volumen de 180000 documentos los fondos contiene toda la documentación administrativa de la JAE y otros documentos personales de miembros de la Generación del 27 y otros protagonistas, manuscritos, cartas etc. El archivo de la JAE se encuentra en buen estado de conservación custodiado en el Centro de Documentación de la Residencia de Estudiantes. Hace unos años y con motivo del centenario de la JAE (2007) se puso a disposición del personal investigador los expedientes de pensión. Para este trabajo, se trata de alumnas y profesoras de la Residencia de Señoritas u otras protagonistas.

La documentación del archivo llegó al Centro organizada en expedientes ordenados alfabéticamente por el nombre de la persona, tanto de las/los solicitantes como de las/los concedidas, por lo tanto, para buscar documentación relativa a las estudiantes y profesoras de la Residencia de Señoritas se puede consultar desde el Portal de la Edad de Plata http://archivojae.edaddeplata.org/ . Ésta aplicación interactiva de consulta de la base de datos permite explorar la actividad de la Junta en los documentos de su archivo. Se accede a la información desde tres entradas: en GEO se navega a través del mapa; RED muestra las relaciones de personas, instituciones y materias; y CRONOS explora la actividad de la JAE a lo largo del tiempo (Residencia de Estudiantes, 2015).

Desde la opción RED se accede a la documentación digitalizada través de algunas variables como "personas y organizaciones" los nombres de las personas. Otras subclases permite acceder a expedientes de instituciones y de materias varias, clasificadas en tres opciones "centros", "instituciones", "otros".

Permite obtener información digital sobre las pensionadas, las especialidades, las instituciones de destino, el tipo de ayuda, las fechas. La aplicación con el término "Residencia de Señoritas" recupera 34 documentos, son una reproducción digital de fichas, cuyo contenido son anotaciones o asientos diarios de gestión económica y organizativa. La documentación reproducida es variada encontrando apuntes sobre acuerdos de arrendamiento de locales, ejecución de obras de reformas, aprobación de presupuestos, autorizaciones varias o nombramientos y destituciones entre otros. En los últimos registros correspondientes a las fichas número 32-33 se hace constar la aceptación de la dimisión de María Maeztu el 21 de septiembre de 1936 debiendo en lo sucesivo prestar servicio en el Escalafón de Escuelas Normales

\subsection{ARCHIVO DE LA RESIDENCIA DE ESTUDIANTES}

La Residencia de Estudiantes transcurridos ya cien años de su creación es una fundación cultural en cuyo patronato se encuentra representado el Centro Superior de Investigaciones Científicas y algunos Ministerios del Gobierno de España. Su Centro de Documentación está integrado por los archivos de la JAE (1907-1936), el Museo Pedagógico Nacional (1982-1936); y los archivos personales Fernando de los Ríos, García Lorca, Luis Cernuda. En cuanto al archivo administrativo original 
de la Residencia de Estudiantes desapareció tras la guerra civil.

El archivo de la Residencia de Estudiantes es un proyecto de rescate de numerosos legados que permanecían dispersos. Contiene los fondos personales de algunos miembros del movimiento literario Generación del 27 y otros protagonistas de la cultura y la ciencia españolas del primer tercio del siglo XX, entre los que se encuentran numerosas cartas, manuscritos, notas autógrafas y abundante documentación gráfica. Y copias microfilmadas de los archivos de Jorge Guillén, Pedro Salinas y Amado Alonso. Este proyecto sigue abierto y promete nuevas incorporaciones. Toda la información está volcada en el catálogo donde se pueden consultar las fichas de descripción del contenido de más de 112000 documentos.

El número de documentos recuperados con el término (Residencia de Señoritas) se reduce a 24, en su mayoría cartas, el expediente de Maeztu y material gráfico.

\subsection{ARCHIVO GENERAL DE LA ADMINISTRACIÓN}

En cuanto a la documentación en el Archivo General de la Administración (AGA), elevada la consulta en los inventarios e instrumentos de descripción pertinentes, nos indica la existencia de expedientes de proyectos de obra: construcciones civiles y monumentos numerosos expedientes desarrollados en la Residencia. Se refirieren a reparaciones y reformas de los pabellones en los varios edificios de la Residencia. En particular, el expediente entre los años 1917/1927 para ampliación y construcción de instalaciones deportivas, entre ellas una piscina y un frontón; además de salas de conferencia, la biblioteca y aulas: y servicios higiénicos. Los proyectos de obras civiles de la Residencia contienen las fechas extremas de 1917 a 1935. Terminada la guerra en 1939 el edificio pasó a llamarse Residencia de Señoritas “Teresa de Cepeda” de Madrid. A propósito del cambio de costumbres que supuso el destino inicial de la Residencia, encontramos en 1941 un proyecto de instalación de capilla y todos los detalles: pila bautismal, altar, comulgatorio, confesionario y mueble de la sacristía entre otros. Finalmente, un proyecto en 1943 para la construcción de un edificio destinado a Escuela-Residencia de bibliotecarias. Todos estos proyectos de obra se pueden consultar en el AGA. Existe un par de expedientes sobre relaciones culturales del Ministerio de Estado y la Embajada de España en París a propósito de un viaje de alumnas acompañadas por Eulalia Lapresta. La documentación del AGA incluye material gráfico, una fotografía de 1933 ilustra la visita del Presidente de la República Niceto Alcalá Zamora al Instituto Escuela, fechada en 1935 sobre el reparto de juguetes en el domicilio de la Residencia Internacional de Señoritas. Identificándose en ella a Josefa Blasco contando unos cuentos a los niños. 


\subsection{ARCHIVO CENTRAL DE LA SECRETARÍA DE ESTADO DE EDUCACIÓN}

Apenas se conserva documentos acerca del Instituto-Escuela, de la Residencia directamente no hay fondos. Encontramos documentación de la asociación de Amigos del Instituto-Escuela, que puede ser interesante, relacionamos los contenidos y signaturas:

-Signatura 122.532. Actos de conmemoración y homenaje, conferencias, discursos y visitas (1976-1992), actos en Mérida y Castellón (1991-1995). archivo de actos de la asociación de Amigos del Instituto-Escuela (1973-1984), archivo de actos de la asociación de Amigos del Instituto-Escuela (1985-2001), excursiones (1979-1988).

-Signatura 122.533. Excursiones, carteles, invitaciones y folletos (1979-2002), asociación de Amigos del Instituto-Escuela de Barcelona (1978-1979), Instituto Escuela de Barcelona (1934-1979).

-Signatura 122.534. Instituto-Escuela. Sección de Alumnos (1923-1940), recopilación de trabajos ejecutados por los alumnos (1924-1925), listas (1977-1987), inscripciones antiguos alumnos domiciliados en el extranjero, instancias al Ministerio de Educación (1939-1941), documentos oficiales (1976-2002), actas de reuniones de la Junta directiva (1983-1988), actas de asambleas del Instituto-Escuela y Junta directiva (1976-2000), antecedentes del Libro Instituto-Escuela (1918-1988) .

-Signatura 122.535. Comunicaciones (1969), correspondencia de entrada y salida de la asociación de Antiguos Alumnos del Instituto-Escuela (1976-1979), correspondencia (1978-1980), cartas comunicaciones y minutas (1981-1989), correspondencia de entrada y salida de la asociación de Antiguos Alumnos del Instituto-Escuela (1977-1997), recortes de prensa (1968-1990), Estatutos de la Asociación de Antiguos Alumnos del Instituto-Escuela, canciones e ilustraciones (1933-1950), publicaciones (1979-1995).

-Signatura 122.536. Publicaciones (1928-1989), Instituto SosBaynat de Castellón (1984-1996), jornadas pedagógicas (1987).

-Signatura 122.537. Cintas grabadas de la asociación de Antiguos Alumnos del Instituto-Escuela (1978-1985), fotografías y microfilm (1924-1983). 


\section{CONCLUSIONES}

El archivo histórico de la Residencia de Señoritas es la principal fuente de documentación para la investigación de la institución y la digitalización documental representa una oportunidad que proporciona ventajas para la consulta de los fondos documentales. El estudio de la serie Correspondencia sirve también para documentar la trascendencia y proyección internacional de la Residencia, para conocer las mentalidades de las residentes y su entorno. Además dar cuenta de las actividades para la emancipación de las mujeres y la libertad de la humanidad, que se desenvolvía en la época.

Actualmente gracias en parte a la digitalización de la documentación y los estudios para la recuperación de la memoria histórica de las mujeres se tienen nuevos datos. Gracias al estudio, se constata una necesaria revisión de la descripción de las series. En realidad hay un evidente sesgo masculino en la clasificación de "Notables". Se consultaron documentos incluidos en la serie correspondencia ALV-ZW, por ejemplo, manuscritos de mujeres protagonistas en la historia en sus ámbitos de acción, feminismo, ciencia, cultura y política. Por ejemplo Francesca Bonnemaison, Luisa Cuesta, Matilde Huici, Margarita de Mayo Izarra, María Sánchez Arbós o instituciones como Lyceum Club. Por lo que recomendamos continuar el proyecto de descripción, especialmente de la Serie ALV-ZW desde una perspectiva que libere y corrija el sesgo de género.

El archivo de la Residencia es una fuente de información para la investigación en general y estudios bibliográficos,estudios de las élites intelectuales y en particular para la historia de las mujeres y del feminismo. En todo caso, se precisa una mejora en la descripción archivística, que haga atractiva la apertura de nuevas líneas de investigación desde varias perspectivas relacionadas con el feminismo, con la trayectoria de la institución en los procesos de transformación, con la memoria democrática, la memoria histórica de las mujeresyla historia social de la escritura.En definitiva, el archivo es una fuente de información de inestimable valor.

\section{AGRADECIMIENTOS}

El presente trabajo está fundamentado en la presentación de una ponencia presentada en la Jornada organizada por el Instituto Universitario de Estudios de Género de la Universidad Carlos III de Madrid y la Asociación Estudiantil Carlos Marx. "Mujeres, educación y cultura. Centenario de la Residencia de Señoritas". Celebrado en Madrid (Campus de Getafe) el 17 de marzo de 2015. Agradezco a la organización de la jornada, a Rosa San Segundo su invitación para presentar este trabajo. A Marisa Mediavilla de la Biblioteca de Mujeres de Madrid por sus afortunados comentarios. A la Fundación Ortega y Marañón por las estimables facilidades para investigar en el archivo. 


\title{
9 FUENTES Y REFERENCIAS BIBLIOGRÁFICAS
}

\author{
ARCHIVOS \\ Archivo de la Residencia de Señoritas \\ Archivo de la Junta de Ampliación de Estudios \\ $<$ http://archivojae.edaddeplata.org/jae_app/JaeMain.html> \\ Archivo de la Residencia de Estudiantes \\ < http://www.edaddeplata.org/edaddeplata/Archivo/archivo/buscador.jsp> \\ Archivo General de la Administración \\ Archivo Central de Educación
}

\section{REFERENCIAS}

AGUILERA SASTRE, Juan (2011). "Las Fundadoras del Lyceum Club femenino español”, en Brecar, 35, 65-90. $<$ https://publicaciones.unirioja.es/ojs/index.php/brocar/article/view/1595/1492> . [Consulta 25/05/2015]

ALCALÁ CORTIJO, Paloma et alt. (2009). Ni tontas ni locas: las intelectuales en el Madrid del primer tercio del siglo XX. [Madrid]: Fundación Española para la $\begin{array}{llll}\text { Ciencia } & \mathrm{y} & \text { Tecnología, } & \end{array}$ $<$ http://www.inmujer.gob.es/publicacioneselectronicas/documentacion/Docume ntos/DE0223.pdf >. [Consulta: 17/02/2015]

BRANCIFORTE, Laura (2015) Experiencias plurales del feminismo español en el primer tercio del siglo pasado: un balance de la historiografía reciente”, en Revista de historiografía (RevHisto), 22, 1 [ejemplar dedicado a: Del ayer al mañana. La historiografía de la historia de las mujeres, del género y del feminism],

235-254.<http://e-revistas.uc3m.es/index.php/REVHISTO/article/view/2654/14 54>. [Consulta 15/09/2015>

CABRERA CALVO-SOTELO, Mercedes (2008). "La Junta para Ampliación de Estudios y de Investigaciones Científicas: historia y presente”, en Josefina Gómez Mendoza, La Junta para Ampliación de Estudios e Investigaciones Científicas y los académicos de la Historia. Madrid: Real Academia de la Historia, pp. 207-221.

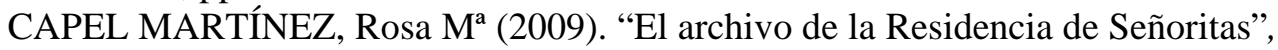
en CEE Participación Educativa, 11, Julio, pp. 156-161.

CODINA-CANET, María Adelina; SAN SEGUNDO, Rosa (2015). "Propuesta de un Centro de Archivo del Feminismo tras el análisis de los fondos documentales del Movimiento Feminista”, en Revista Española de Documentación Científica (admitido para su publicación en la REDC).

CONNELLY, Joan (1982). "La enseñanza superior de la mujer en España: 
relaciones entre universitarias españolas y estadounidense, 1877-1980, en Nuevas perspectivas sobre la mujer. Actas de las primeras Jornadas de investigación interdisciplinaria. Madrid: Universidad Autónoma, Seminario de Estudios de la Mujer, pp. 196-205.

EIROA, Matilde (2015). 'La popularización del saber y la "generación de las modernas": revistas y espacios femeninos en la España de entreguerras", en Ammis, pp. 1-6.<http://amnis.revues.org/2621http://amnis.revues.org/2621>. [Consulta: 17/02/2015]

EGOSCOZÁBAL CARRASCO, Pilar; MEDIAVILLA HERREROS, María Luisa (2012). La bibliotecaria Luisa Cuesta Gutiérrez (1892-1962). Revista General de Información $y \quad$ Documentación. Vol. $22 \quad$ (2012) 169-187.<http://dx.doi.org/10.5209/rev_RGID.2012.v22.39655>. [Consulta: 21/02/2014]

FAGOAGA, Concha (1985), La voz y el voto de las mujeres. 1877-1931. Barcelona: Icaria, pp. 214.

- (2002). "El Lyceum Club de Madrid, elite latente”, en DanièleBussyGenevois (ed.), Les espagnolesdansl'histoire. Une socialiblitédémocratique (XIX -XX siècles). Saint-Denis: PressesUniversitaires de Vincennes, pp. 145-167.

GÓMEZ BLESA, Mercedes (2015). Las Sinsombrero, Sin ellas la historia no está completa (Documental Online).Madrid: RTVE. <http://www.rtve.es/lassinsombrero/es/webdoc/>. [Consultado 2/07/2015]

HURTADO, Amparo (1999), "El Lyceum Club Femenino (Madrid, 1926-1939)”, en Boletín de la Institución Libre de Enseñanza, II época, 36, pp. 23-36.Junta de Ampliación de Estudios (1916), en Memorias de la JAE. 1914-1915. Madrid.

KIRKPATRICK, Susan (2003). Mujer, modernismo y vanguardia en España (1898-1931). Madrid: Cátedra, pp. 322.

LÓPEZ COBO, Azucena. ; BASABE, Nere (2007). "La residencia de señoritas. La contribución de la JAE a la educación de la mujer. Despejo y disposición. La educación de la mujer española entre dos siglos”. Circunstancia, Año V, 14-septiembre.<http://www.ortegaygasset.edu/publicaciones/circunstancia/anov---n--14---septiembre-2007/investigaciones-en-curso\#6>. [Consulta $12 / 06 / 2015]$

MAGALLÓN PORTOLÉS, Carmen (2004). Pioneras Españolas en las ciencias. Las mujeres del Instituto Nacional de Física y Química. Madrid: Consejo Superior de Investigaciones Científicas. $<$ http://www.jae2010.csic.es/documentos/publicaciones/publicacion_pioneras.p df $>$. [Consulta: 10/03/2015]

MAILLARD, María Luisa (1990). Asociación Española de Mujeres Universitarias (1920-1990). Madrid: Asociación Española de Mujeres Universitarias e Instituto de la Mujer, pp. 163.

MANGINI, Shirley (2001). Las modernas de Madrid: las grandes intelectuales españolas de la vanguardia. Barcelona: Península, pp. 272. 
-(2006). "El Lyceum Club de Madrid: un refugio feminista en una capital hostil”, en Asparkía, 17, pp. 125-140.

MARINA, José Antonio; RODRÍGUEZ DE CASTRO, María Teresa (2009). La conspiración de las lectoras. Barcelona: Anagrama, pp. 274.Memorias de la JAE. 1914-1915. Madrid, 1916, pp. 300.

MORENO Y CASANOVA, Juan José (2001). "Archivo y biblioteca de la Asociación para la Enseñanza de la Mujer”, en Fernando de Castro y su legado intelectual. Madrid: Fundación Fernando de Castro, pp. 197-205.

NASH, Mary (2008). "Mujeres, conciencia de género y movilizaciones sociales”, en Rosa María Capel Martínez. Cien años trabajando por la igualdad. Madrid: Fundación Francisco Largo Caballero: Instituto de la Mujer, pp. 117-130.

NÚÑEZ PUENTE, Sonia (2008). "Dos cartas inéditas de María Lejárraga dirigidas a Gregorio Martínez Sierra”, en UNED. Revista Signa, 17,pp. 283-291.

PAU, Antonio (2001). "Ideas y empresas pedagógicas de Fernando de Castro”, en Fernando de Castro y su legado intelectual. Madrid: Fundación Fernando de Castro, pp. 119-121.

PÉREZ-VILLANUEVA TOVAR, Isabel (2011). La residencia de estudiantes, 1910-1936; grupo universitario y residencia de señoritas. Madrid: Acción Cultural Española [etc.], pp.796.

-(1990). La residencia de estudiantes; grupos universitarios y de señoritas, Madrid 1910-1936. Madrid: Centro de Publicaciones, Ministerio de Educación y Ciencia, $<$ https://sede.educacion.gob.es/publiventa/detalle.action?cod=1413>.

pp.389.

[Consulta: 25/02/2015]

-(1989). María de Maeztu: una mujer en el reformismo educativo español. Madrid: Universidad Nacional de Educación a Distancia, pp. 220.

POZO ANDRÉS, M ${ }^{a}$ del Mar (1985), “Actividades culturales y pedagógicas del Lyceum Club Femenino de Madrid (1926-1936)”, en Ángeles Galino Carrillo, La educación en la España contemporánea, cuestiones históricas. Libro homenaje a Ángeles Galino. Madrid: Sociedad Española de Pedagogía, pp. 203-212.

"Residencia de Estudiantes", Archivo de la JAE. $<$ http://archivojae.edaddeplata.org/jae_app>. [Consulta: 25/02/2015]

RIBAGORDA, Álvaro (2009). Caminos de la modernidad: espacios e instituciones culturales de la Edad de Plata (1898-1936). Madrid: Biblioteca Nueva. Fundación Ortega y Gasset, pp. 289.

SAN SEGUNDO, Rosa; CODINA-CANET, María Adelina. "Incidencia de aspectos historiográficos en la organización de los saberes en la universidad", en BrazilianJournal of InformationScience, 8, 1-2. $<$ http://dialnet.unirioja.es/descarga/articulo/4972285.pdf $>$ [Consulta 20/02/2015]

SANTALLA LÓPEZ, Manuela (1994). "La condición femenina en Concepción 
Arenal”, en Arenal. Revista de Historia de las mujeres, 1 (1), pp. 103-115.

SIMÓN PALMER, María del Carmen (2006). "Mil estudios actuales sobre escritoras del siglo XX”, en Arbor, 721, septiembre-octubre, pp. 661-793. $<$ http://arbor.revistas.csic.es/index.php/arbor/article/view/60/60>.[Consulta: 25/02/2015]

VÁZQUEZ RAMIL, Raquel (2012). Mujeres y educación en la España contemporánea: La Institución Libre de Enseñanza y la residencia de Señoritas. Madrid: Akal, pp. 494.

ZULUETA, Carmen de; MORENO, Alicia (1993). Ni convento ni college: la Residencia de señoritas. Madrid: Publicaciones de la residencia de Estudiantes, pp. 267.

ZULUETA, Carmen de (2002). Instituto Internacional en Madrid: primer centenario. Barcelona; Madrid: Lunwerg, pp. 53. 


\section{ANEXO}

Índice topográfico del Archivo

Caja 1. Invitaciones-anuncios

Caja 2. Carteles y folletos

Caja 3. Folletos de la Residencia de Estudiantes y del Centro de Estudios Históricos

Caja 4. Biblioteca

Caja 5. Borradores de Memorias dirigidas a la Junta de Ampliación de Estudios

Caja s 6 y 7. Asociación Alumnas

Caja 8. Correspondencia de la Junta de Ampliación de Estudios

Caja 9. Comité Boston

Caja 10. Asociaciones de mujeres y otras asociaciones

Caja 11. Clases. (Años 1920-1933)

Caja 12. Clases. (Años 1933-1936)

Caja 13. Biblioteconomía, clases de

Caja 14. Anuncios. (Años 1920-1935)

Caja 15. Enfermería

Cajas 16 y 17. Régimen interior

Caja 18. Inventarios, documentación de los tablones de anuncios, régimen interno, coros, servicios médicos, actos culturales, conferencias, y clases, biblioteca, excursiones, revistas y periódicos, turnos de guardia. (Años 1920-1935)

Caja 19 Correspondencia de María de Maeztu. (Años 1919-1933)

Cajas 20-21 Correspondencia Notables

Cajas 22-48 Correspondencia AB - ALV,ALV - AZ ,BAC - BAS ,BAS - BORR ,BORR CAR , CAR - CLA , CLA - CUY , CHA - DUT , EA - FERN ,FERN - FY , GAB - GAS ,GAS - GW , HAD - IZ , JAC - CKY, LAB - LOW , LOW - MAR, MAR - MON ,MON - NY , OBE - PELL ,PELT - QUI , RAG -RIU , RIU -RUB ,SAA -SAN ,SANZ -SIM , SIM -SW , TAB -VALL , VALL -ZW

Cajas 49-59 Correspondencia ordenada (Años 1920-1936)

Cajas 60 Correspondencia, gastos y otros . (Años 1935-1939 Valencia)

Cajas 61 Excursiones y fiestas

Cajas 62-64 Expedientes

Cajas 65-66 Edificios

Caja 67 Presupuestos

Caja 68 Balances

Caja 69 Nóminas

Caja 70 Años 1920-1927 Facturas sueltas

Cajas 71-92 Carpetas contabilidad. (Años 1928-1935)

Cajas 93-100 Fotocopias y documentación miscelánea: Cartas de Soledad María de Maeztu, María Lezarraba, María Lacampra. Notas correspondencia (archivo), Instituto Escuela, Papeles inventario. Archivo Bullón Eloy, Maeztu - Ortega. Revista Residencia incompleta, Documentación sobre María de Maeztu donada por Carmen Zulueta 1993. Residencia: cursos y conferencias, folletos, Cincuentenario, libros. 